Anaesthesist 2022 $\cdot 71: 311-317$

https://doi.org/10.1007/s00101-021-01066-8

Angenommen: 11. Oktober 2021

Online publiziert: 6. Dezember 2021

(c) The Author(s), under exclusive licence to Springer Medizin Verlag $\mathrm{GmbH}$, ein Teil von Springer Nature 2021
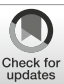

\section{Organspende - Nicht nur eine intensivmedizinische Aufgabe}

\author{
A. Rand $\cdot$ T. Koch $\cdot$ M. Ragaller \\ Klinik und Poliklinik für Anästhesiologie und Intensivtherapie, Universitätsklinikum Carl Gustav Carus, \\ Dresden, Deutschland
} für ein Spenderorgan standen. Sowohl im Eurotransplant-Verbund als auch weltweit gehört Deutschland mit 10,8 Organspendern/Mio. Einwohner im Jahr 2019 zur Schlussgruppe. Sämtliche politischen Versuche, die Spenderzahlen zu steigern, scheinen bislang ohne Effekt geblieben zu sein. Darüber hinaus hat die durch das "severe acute respiratory syndrome coronavirus 2" (SARS-CoV-2) ausgelöste Pandemie zum weiteren Rückgang der Spenderzahlen geführt. Der Intensivmedizinerin kommt im Prozess der Erkennung möglicher Spender sowie als erste Ansprechpartnerin für die Angehörigen eine zentrale Rolle zu. Jedoch existieren nicht nur in den gesellschaftlichen und medialen Diskussionen um das Thema Organspende viele Unklarheiten, sondern auch bei den intensivmedizinisch tätigen Ärzten. Viele Annahmen und Hypothesen, die mit den niedrigen Spenderzahlen in einen Zusammenhang gebracht werden, lassen sich wissenschaftlich jedoch nicht belegen und sollen in diesem Beitrag diskutiert werden.

\title{
Schlüsselwörter
}

Transplantation · Hirntod · Herztod · Religiöser Glauben · Mutmaßliche Einwilligung

\section{Einführung}

Die zwingende Voraussetzung für die Organentnahme ist die Feststellung des irreversiblen Hirnfunktionsausfalls (IHA, ehemals „Hirntod“) nach der Richtlinie der Bundesärztekammer (BÄK) [29].

Aufgrund der für den Ausfall ursächlichen Hirnschädigung ist davon auszugehen, dass sich ein Großteil der Patientinnen bereits vor der Diagnostik und Feststellung in intensivmedizinischer Behandlung befunden haben muss.

Traumatisch bedingte Hirnschädigungen treten z. B. im Rahmen eines Polytraumas zusammen mit anderen potenziell tödlichen Verletzungen auf. Entwickeln diese Patienten im klinischen Verlauf weitere Organdysfunktionssyndrome, wird fälschlicherweise häufig eine generelle Untauglichkeit für die Organspende im Fall eines IHA angenommen. Darüber hinaus können evtl. Therapielimitierungen auf dem Boden eines mutmaßlichen oder tatsächlich geäußerten Patientenwillens eine überaus komplexe und schwierige klinische Gesamtkonstellation entstehen lassen. Schlussendlich steht am Ende der Therapiepfade, die aus diesen Konstellationen gebildet werden, zwar häufig das Versterben des Patienten, jedoch nicht die erfolgreiche Organspende.

\section{Analyse der Spendersituation in Deutschland}

Die Allokation der in Deutschland explantierten Organe wird durch die Deutsche Stiftung Organtransplantation (DSO) über die Organisation Eurotransplant mit Sitz in Leiden, Niederlande, durchgeführt. Eurotransplant koordiniert die Organvermittlung aus den Staaten Belgien, Deutschland, Kroatien, Luxemburg, Niederlande, Österreich, Slowenien und Ungarn. Obwohl Deutschland mit ca. 83 Mio. Einwohnerinnen das mit Abstand größte Mitgliedsland ist, weist es seit Jahren im Vergleich die 


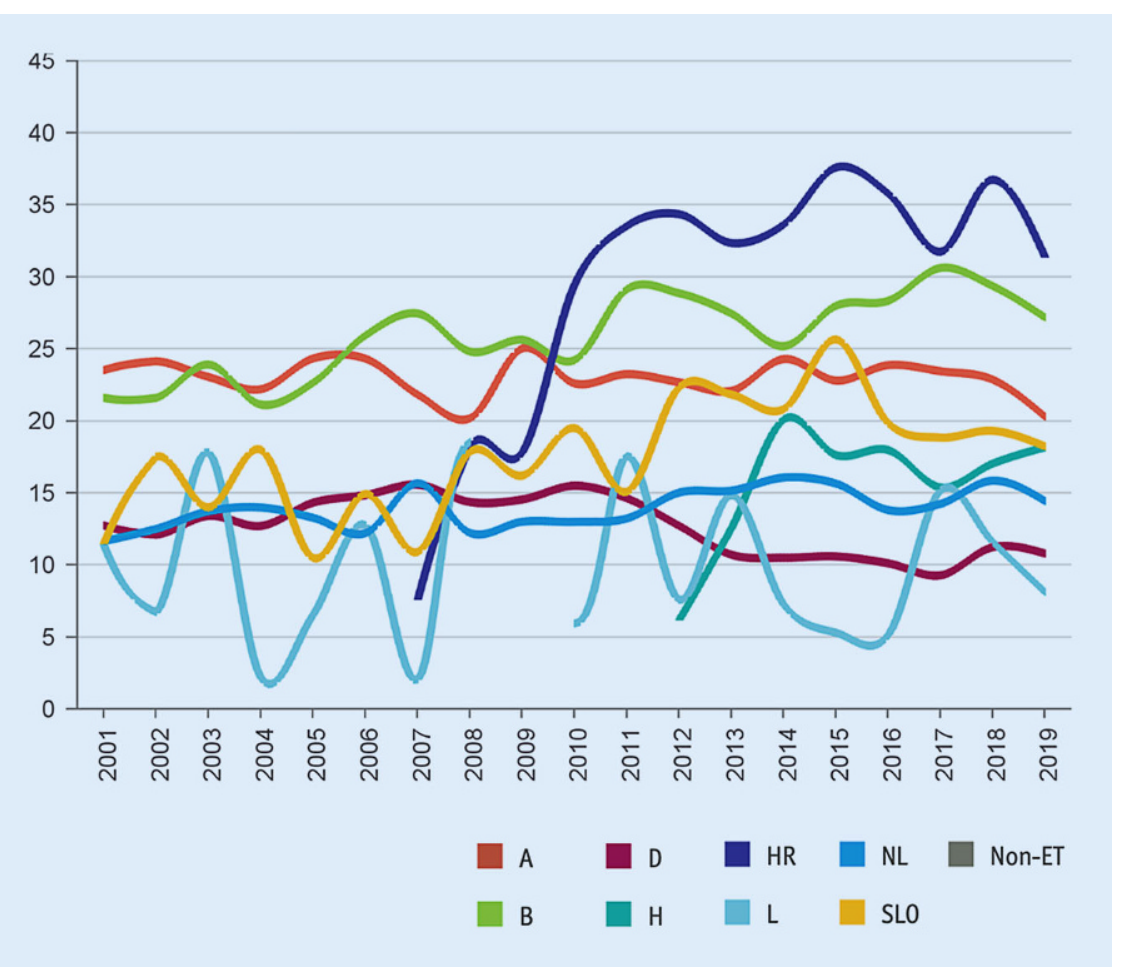

Abb. $1 \Delta$ Organspender pro Mio. Einwohner im Eurotransplant-Verbund. A Österreich, D Deutschland, $H R$ Kroatien, NL Niederlande, B Belgien, $H$ Ungarn, $L$ Luxemburg, SLO Slowenien, Non- ET Länder außerhalb des Verbundes, mit denen Eurotransplant Abkommen zur Organallokation hat. (Aus Eurotransplant International Foundation [14], @ all rights reserved, mit freundlicher Genehmigung)

niedrigsten Spenderzahlen pro Million Einwohnerinnen auf (• Abb. 1). Gleichzeitig standen am 31.12.20199271 Patienten auf der Warteliste für ein vermittlungspflichtiges Organ (Leber, Herz, Lunge Pankreas, Darm und Niere).

Während in anderen europäischen Ländern die Spendezahlen nach der Einführung von Programmen und Gesetzesänderungen gesteigert werden konnten, gingen sie in Deutschland trotz einiger politischer Anstrengung weiter zurück [32]. Sowohl in der Allgemeinbevölkerung als auch in Fachkreisen zirkulieren verschiedene Theorien über Gründe, die für die niedrigen Spenderraten verantwortlich sein sollen und nicht alle sind mit wissenschaftlicher Evidenz untermauert. Der vorliegende Beitrag widmet sich der Aufarbeitung und Analyse einiger der Annahmen, die im Folgenden dargestellt sind.

\section{Verbesserte Unfallverhütung und Sicherheitstechnologien}

Theorie: Durch verbesserte Unfallverhütung in der Arbeitswelt und Sicherheits- technologien, z.B. im Straßenverkehr, kommt es zu einem Rückgang der Inzidenz schwerer, insbesondere traumatischer Hirnschäden und damit potenzieller Spenderinnen [34].

Im Jahr 2019 waren nur bei 13,1\% der Patientinnen, die in Deutschland zu Organspenderinnen wurden, die Ursache für die Hirnschädigung in einem Schädel-HirnTrauma (SHT) begründet. Das SHT steht lediglich an 3. Stelle der Ursachen für eine Hirnschädigung, nach ischämisch-hypoxischen Hirnschäden (23,3\%) und intrakraniellen Blutungen (53,3\%) [12]. Weiss et al. verglichen die Spenderinnenraten und die Letalität nach traumatischen und nichttraumatischen Hirnschäden in 28 Staaten über einen Zeitraum von 15 Jahren und konnten zeigen, dass die Letalität im Beobachtungszeitraum deutlich zurückgeht (in Deutschland um 20\%). Darüber hinaus haben Fortschritte in der aktuellen Schlaganfallbehandlung wie etwa die flächendeckende Einführung von Stroke Units und die bessere Früherkennung die Zahl der malignen Infarkte und damit auch die Zahl der potenziellen Spendenden weiter redu- ziert [27]. Der zu erwartende Rückgang in den Spenderzahlen blieb jedoch in den meisten Ländern aus. Im Gegenteil konnten die meisten Staaten die Spenderkonversion, also den Anteil an Spendenden aus dem Gesamtkollektiv der Verstorbenen erhöhen, mit Ausnahme Deutschlands [34].

\section{Einstellungen der Bevölkerung zur Organspende}

Theorie: Die Zustimmung in der Bevölkerung zum Thema Organspende ist mangelhaft und durch die "Organspendeskandale" nachhaltig geschädigt.

Im Jahr 2012 wurde aufgedeckt, dass an mehreren deutschen Transplantationszentren systematisch Laborparameter verändert wurden, um den Patientinnen der eigenen Zentren einen Vorteil bei der Organallokation zu verschaffen. Die breite mediale Rezeption weniger negativer Beispiele im Kontrast zur quasi nichtvorhandenen Berichterstattung vieler positiver Auswirkungen des Transplantationswesens in Deutschland wird für die Entstehung einer misstrauischen Haltung in der Bevölkerung und daraus resultierend sinkende Spenderzahlen mitverantwortlich gemacht [21]. Zwar hat sich der Anteil der Menschen mit einer „eher negativen“ Einstellung zur Organspende seit 2010 von 5 auf $8 \%$ fast verdoppelt, jedoch ist parallel der Anteil der "eher positiv" eingestellten Menschen von 79 auf $84 \%$ kontinuierlich gestiegen. Gleichzeitig besitzen deutlich mehr Menschen einen Organspendeausweis (2010: $25 \%, 2018: 36 \%$ ), und immer mehr dokumentieren ihre Entscheidung zur Organspende in einem Ausweis oder einer Patientenverfügung (PV; 2012: 22\%, 2018: 39\%, [9]).

In den per Organspendeausweis dokumentierten Entscheidungen wird nur in $4 \%$ der Fälle einer Organentnahme widersprochen (15\% bei Dokumentation in PV). Die häufigsten Begründungen sind, dass die Patientinnen sich oder ihre Organe als zu alt oder zu krank empfinden (ca. 24\%), und das Misstrauen gegenüber dem System der IHA-Diagnostik und Allokation (ca. 22\%, [9]). Ob bei Erstgenanntem tatsächlich eine absolute oder relative Kontraindikation für eine Organspende 
vorliegt, ist unbekannt, aber als eher unwahrscheinlich anzusehen.

\section{Religiöser Glaube und ethische Grundsätze}

Theorie: Religiöse Gründe verbieten eine Organspende oder akzeptieren die Definition des IHA nicht [6].

Religiöse und ethische Gründe werden zwar seltener als Ablehnung einer Organspende genannt, kommen jedoch vor. In dokumentierten Entscheidungen sind sie der dritthäufigste Grund eines Widerspruchs (ca. 16\%) [9]. Spezifische Argumente gegen Organspende oder den IHA finden sich jedoch bei keiner der monotheistischen Religionen.

- Die christlichen Kirchen in Deutschland betrachten die Organspende als „Akt der Nächstenliebe und Solidarität über den Tod hinaus" [17].

- Die muslimische Rechtsprechung erlaubt bereits seit den 1950er-Jahren die Transplantation von Organen und Geweben. Im Jahr 1986 wurde auf der 3rd International Conference of Islamic Jurists die Gleichsetzung von Herzund Hirntod nach islamischem Recht anerkannt [2].

- Das Oberrabbinat Israels erkannte ebenfalls 1986 die Gültigkeit des Hirntods an. Seit 2008 ist der „BrainRespiratory Death Act" offizielles jüdisches Recht [5]. Während es nach jüdischem Recht eigentlich verboten ist, den Körper eines Verstorbenen zu verändern, sehen es viele Gelehrte als statthaft an, wenn dies in der Intention geschieht, einen anderen Menschen zu retten [1]. Nichtsdestotrotz scheint das Thema Organspende insbesondere unter Angehörigen orthodoxer Glaubensgemeinschaften weiterhin sehr restriktiv gehandhabt zu werden [5].

Die Stellung anderer Religionen zur Organspende, wie Hinduismus, Buddhismus und Sikh, werden detailliert in einer Arbeit der Universität von Cardiff, Wales, Vereinigtes Königreich Großbritannien und Nordirland, behandelt [6].

\section{Entscheidungs- vs. Widerspruchs- lösung}

Theorie: Durch die Entscheidungslösung entfallen viele potenzielle Spender, da sie einer Spende zu Lebzeiten zustimmen müssen. Eine Widerspruchslösung würde mehr Spenderinnen generieren [13].

In Deutschland wird die erweiterte Zustimmungsregelung („opt-in“) zur Organspende praktiziert, die aktuell als Entscheidungslösung deklariert und im $\$ 2$ Abs 2 des Transplantationsgesetzes (TPG) festgeschrieben ist [18]. Zuletzt votierte im Januar 2020 der Bundestag im Rahmen der Novellierung des TPG gegen eine Gesetzesänderung hin zu einer Widerspruchslösung („opt-out"). Die Bürgerin soll sich zu Lebzeiten mit dem Thema Organspende auseinandersetzen, eine persönliche, freie Entscheidung fällen und diese dokumentieren. Damit sich mehr Menschen ausführlicher mit dem Thema befassen und ihre Entscheidung dokumentieren, sollen ab 2022 auch Hausärzte regelmäßig über das Thema aufklären und Informationsmaterial aushändigen. Im Vorfeld der erneuten Diskussion des TPG hatte sich u.a. der 121. Deutsche Ärztetag 2018 in Erfurt klar für die Widerspruchslösung positioniert.

Wissenschaftlich konnte die Frage, ob eine Opt-in- einer Opt-out-Lösung bezüglich der Organspendezahlen überlegen ist, bislang nicht eindeutig geklärt werden. Rithalia et al. konnten 2008 in einer Übersichtsarbeit mehrere Studien identifizieren, die einen signifikanten Anstieg der Spenderzahlen nach Einführung einer Widerspruchslösung berichteten. Trotzdem wiesen die Autoren darauf hin, dass z.B. auch andere Faktoren, die nicht in den Studien untersucht worden waren, sich aber zeitgleich in den Beobachtungszeiträumen positiv entwickelt hatten, die Änderung verursacht haben könnten. Beispielhaft soll die gesellschaftliche Zustimmung zur Organspende genannt sein [30]. Eine aktuellere Analyse von 35 Ländern, bei denen 17 Länder eine Optout- und 18 Länder eine Opt-in-Regelung verfolgten, konnte nur eine nichtsignifikant höhere Spenderrate für Länder mit Opt-out-Regelungen zeigen. Die Autoren schlussfolgerten, dass allein die Änderung des Zustimmungsformates keine
Lösung für die nachhaltige Steigerung der Spenderzahlen ist [4].

\section{Identifikation potenzieller Spender im Krankenhaus}

Theorie: Die Identifikation potenzieller Spender im Krankenhaus funktioniert schlecht oder ist aufgrund der Seltenheit für die einzelne Intensivstation ein eher "unbekannter Prozess", weshalb eine groBe Anzahl der potenziellen Spenderinnen vor der Hirntoddiagnostik verstirbt.

Wie bereits eingangs erwähnt, liegt die Verantwortung für die Spendererkennung und das folgende Prozedere bei der Intensivmedizinerin. Es besteht jedoch Grund zu der Annahme, dass diese Erkennung in der heterogenen deutschen Krankenhauslandschaft nur unzureichend funktioniert und aufgrund mangelnder Erfahrung Wissenslücken bestehen [15].

Seit 2010 kann mit dem Programm „DSO-Transplantcheck für Excel" retrospektiv aus den §21-Daten eines Krankenhauses gezielt nach Patientinnen gesucht werden, die potenziell für eine Organspende geeignet gewesen wären. Das Programm sucht nach Todesfällen, bei denen während des Krankenhausaufenthalts der betroffenen Patientinnen mindestens eine Diagnose einer primären oder sekundären Hirnschädigung, die Durchführung einer maschinellen Beatmung sowie keine Diagnose einer Kontraindikation verschlüsselt wurde. Diese Fälle bilden die "Grundgesamtheit des Spenderpotenzials". Ein Pilotprojekt im Auftrag der DSO, die "In-house"-Koordination in 112 deutschen Krankenhäusern, zeigte, dass in den betrachteten Kliniken zwischen 2010 und 2012 zwar ein Großteil der möglichen Spenderinnen erfasst, jedoch bei insgesamt 411 Fällen keine IHA eingeleitet wurde, obwohl die retrospektive Analyse dies als sinnvoll erachtete. Zwar entsprechen diese 411 Fälle nur 1,8\% aller mit einer Hirnschädigung Verstorbenen, jedoch hätten sie zu einer Steigerung der Spendezahlen um $31 \%$ geführt, wenn bei allen eine Spende realisiert worden wäre [7]. Bereits damals wurden Forderungen nach strukturellen Verbesserungen der Spenderinnenerkennung, Schulung der beteiligten Mitarbeiterinnen und nach einer besseren Aufwandsvergütung für 
Tab. 1 Maastricht-Kriterien für die Einteilung von "donators after cardiac death"

\begin{tabular}{|l|l|}
\hline Kategorie & Kriterium \\
\hline I & Herzstillstand bei Krankenhausaufnahme ohne erfolgte Reanimation \\
\hline II & Herzstillstand nach frustraner Reanimation \\
\hline III & Erwarteter Herzstillstand, z. B. nach Therapiebeendigung \\
\hline IV & Unerwarteter Herzstillstand nach Diagnosestellung des Hirntods \\
\hline
\end{tabular}

die Krankenhäuser zur Ausschöpfung des Spenderpotenzials formuliert.

Eine weitere deutschlandweite Analyse aller 112.172.896 stationären Fälle zeigte von 2010 bis 2015 einen Anstieg des Spenderpotenzials um 13,9\%. Im gleichen Zeitraum gingen jedoch sowohl der Anteil der DSO-Kontaktaufnahmen für potenzielle Spenderinnen, der Anteil der tatsächlich realisierten Spenden sowie das Verhältnis von Spenden zu DSO-Kontaktaufnahmen zurück [31]. Brauer et al. analysierten nach der oben beschriebenen Methode die Todesfälle in den Krankenhäusern der DSO-Region Ost aus dem Jahr 2016. Es wurden 2442 Todesfälle einer Einzelfallanalyse unterzogen, wobei 73 zusätzliche potenzielle Spender identifiziert wurden, bei denen auf der Grundlage der Analyse eine IHA-Diagnostik indiziert gewesen wäre, aber nicht durchgeführt worden war. Nach Abzug der Wahrscheinlichkeit für einen nichtdiagnostizierbaren IHA und der durchschnittlichen Ablehnungsquote verblieben 24 zusätzliche, unerkannte potenzielle Spenderinnen [8].

Die BÄK hat zur Unterstützung der Spenderinnenerkennung eine eigene Richtlinie publiziert [28].

Unterstützung bei der Erkennung von Patienten mit möglicherweise bevorstehendem IHA können auch Abfrage-Tools in Patientendatenmanagementsystemen (PDMS) leisten. Durch eine automatisierte Meldung von Patienten, die mögliche Symptome eines IHA aufweisen, an die Transplantationsbeauftragten (TxB) konnte die Zahl nichterkannter potenzieller IHA-Fälle deutlich reduziert werden [33].

\section{Thematisierung der Organspende in Patientenverfügungen}

Theorie: In vielen PV wird sich nicht oder nur unzureichend zum Thema Organspende oder bezüglich der Fortführung einer intensivmedizinischen Therapie zur Realisierung einer Organspende geäu-
Bert. Dies kann im klinischen Alltag zu Therapielimitierungen oder -beendigungen vor Beginn einer IHA-Diagnostik führen [19].

Immer mehr Menschen in Deutschland besitzen eine PV.Jedoch wird sich nur in der Hälfte von diesen überhaupt zur Organspende geäußert [9], bzw. wird die Frage nach der Organspende in den entsprechenden Vordrucken nicht gestellt. Dies birgt das substanzielle Risiko, dass die Intensivtherapie im Fall einer infausten oder nicht dem Willen der Patientinnen entsprechende Prognose, beendet wird, ohne die Möglichkeit einer Organspende in Betracht zu ziehen. Dies gilt insbesondere deshalb, weil dies in den meisten Fällen mit einer zumindest zeitlich begrenzten Fortführung oder gar Neuaufnahme intensivmedizinischer Maßnahmen verbunden wäre.

Im Jahr 2019 wurden $40 \%$ der Entscheidungen für oder gegen eine Organspende anhand des mutmaßlichen Willens der Patientinnen gefasst. In den Fällen, in denen keine Zustimmung erfolgte, war die mangelnde Kenntnis der Einstellung des Verstorbenen die zweithäufigste Ursache [12]. In einer weiteren Analyse von Wesslau et al. kannten sogar $90 \%$ der Angehörigen den Willen des Verstorbenen nicht [35]. Im Prinzip wäre diese Problematik durch die Aufnahme einer Frage nach der Bereitschaft zur Organspende zu lösen, wie sie beispielhaft in die Vorlage der PV der Sächsischen Landesärztekammer implementiert ist. Im Zustimmungsfall ergäbe sich dann die Möglichkeit, die intensivmedizinische Therapie bis zum IHA resp. bis zur Organentnahme fortzusetzen, da dies in völliger Übereinstimmung mit dem Patientinnenwillen geschähe. Die BÄK hat in einem Arbeitspapier versucht, den Umgang mit PV im Kontext einer Organspende zu klarifizieren [3].

Das Konzept des Beginns bzw. der Fortführung intensivmedizinischer Maßnahmen im Sinne eines Erhalts der Organfunktion spendewilliger Patientinnen, bei denen das Eintreten des Hirntodes in absehbarer Zeit zu erwarten ist, wird unter dem Begriff „intensive care to facilitate organ donation" (ICOD) in mehreren europäischen Ländern praktiziert [24]. In Spanien wurden 2014-2015 24\% der gesamten Spenden durch ICOD ermöglicht [11].

\section{Erweiterung des potenziellen Spenderinnenkreises um sog. Herztote}

Theorie: Die Voraussetzungen zur Diagnose des IHA und die Durchführung einer IHA-Diagnostik sind zu komplex und treten in vielen Szenarien nicht ein. Eine Erweiterung des Kreises potenzieller Spenderinnen um Herztote würde die Spenderrate möglicherweise deutlich erhöhen.

In Deutschland ist nach §3 Satz 2 TPG die Entnahme von Organen und Geweben toter Spender nur zulässig, nachdem der endgültige, nichtbehebbare Ausfall der Gesamtfunktion des Großhirns, des Kleinhirns und des Hirnstamms (IHA) festgestellt wurde. Die Verfahrensregeln werden durch die BÄK per Richtlinie festgelegt [29].

Neben den Spenderinnen nach IHA („donation after brain death", DBD) akzeptieren viele Länder auch die sog. herztoten Spenderinnen („,donation after cardiac death" [DCD] oder „non heartbeating donor" [NHBD]). Seit 1995 unterscheiden die Maastricht-Kategorien 4 mögliche DCD (• Tab. 1; [22]).

Die Kategorien I und II werden aufgrund ihres nichtvorhersehbaren Auftretens als unkontrolliert ( $U D C D$ ) sowie Kategorie III als kontrolliert (CDCD) beschrieben. Die Richtlinien der einzelnen Länder sind unterschiedlich. Allen gemeinsam ist jedoch, dass auf den pulslosen Herz-Kreislauf-Stillstand (nicht in allen Ländern muss eine Asystolie vorliegen) eine „no-touch period" von 5-30 min folgt, bevor der Patient für tot erklärt und mit der Organentnahme begonnen werden kann [23]. Da per DCD gespendete Organe vor der Transplantation einer warmen Ischämie ausgesetzt sind, waren die Erfolgsraten und das Transplantatüberleben im Vergleich zu DBD gespendeten Organen häufig schlechter, was durch eine Optimierung des Spendepro- 
zesses jedoch angeglichen werden konnte $[10,16,25]$.

Die BÄK hat seit 1995 in mehreren Stellungnahmen die DCD als Voraussetzung für eine Organentnahme abgelehnt, 2015 hat sich der Deutsche Ethikrat diesem Standpunkt angeschlossen [20]. Grundlage der Argumentation ist die sog. ToteSpender-Regel („dead donor rule“, DDR), nach der eine Organentnahme ausschließlich an toten Spenderinnen erfolgen darf. Die DDR basiert auf dem deontologischen Grundsatz, dass ein menschliches (Weiter-)Leben kein Mittel zum Zweck (einer Organentnahme) sein darf [26]. Die Erweiterung der in Deutschland zulässigen Todeskriterien sichere Todeszeichen, nicht mit dem Leben zu vereinbare Verletzungen und IHA um den "Herztod" würde umfangreiche Gesetzesänderungen voraussetzen. Darüber hinaus ist zu definieren, ab welcher Dauer eines Herz-Kreislauf-Stillstands ein Überleben so unwahrscheinlich ist, dass ein definitiv nichtüberlebensfähiger Zustand aktiv durch die Explantation herbeigeführt werden darf. Somit bleibt letztendlich die Definition vom Tod des Menschen, sowohl im wissenschaftlich-ethischen als auch im gesamtgesellschaftlichen Diskurs, neu zu überdenken und ggf. rechtlich zu verankern, um die DDR nicht zu verletzen.

Dabei gäbe es für CDCD-Spenden theoretisch gute Argumente. Patientinnen, die sich zu Lebzeiten entschlossen haben, Organe zu spenden, und bei denen aufgrund einer schweren Erkrankung eine infauste Prognose oder ein nicht mit ihrem Willen zu vereinbarendes Therapiekonzept bestünde, könnten nach kontrollierter Therapiebeendigung explantiert werden. Die Frage, ob die Hirnfunktion nach einer bestimmten Wartezeit im Anschluss an den Eintritt des Herzstillstands irreversibel erloschen ist, würde sich in diesen Fällen nicht stellen. Denn das Versterben wäre Folge einer bewussten Entscheidung in Ermangelung eines Therapieziels bzw. das konsequente Umsetzen des Patientenwillens und würde nicht auf dem Boden der artifiziellen wissenschaftlich-ethischen Todesdefinition des IHA fußen. Kann medizinisch mit ausreichender Wahrscheinlichkeit kein mit dem Patientinnenwillen zu vereinbarendes Therapieziel mehr erreicht werden, ist die Therapiebeendigung, auch zur Ver- hinderung der Leidensverlängerung, mit der Akzeptanz des konsekutiven Versterbens der Patientin nicht nur Gebot für den Arzt. Spendewilligen Patienten in solch einer Situation die Organspende zu versagen anstatt sie zu ermöglichen, weil diese den „falschen Tod" sterben, ist ethisch zumindest diskutabel.

Das Potenzial, das DCD-Spenderinnen für die Steigerung der Gesamtspenderate haben, ist sehr unterschiedlich. Während der Anteil an DCD-Spenderinnen in Spanien 2015 nur 17\% betrug, waren es in Großbritannien $41,8 \%$ und in den Niederlanden sogar 54,9\% [34].

\section{Resümee}

Die Betrachtung der Organspende als rein intensivmedizinisches Thema stellt einen eindimensionalen Blickwinkel dar und würde dem Problem des drängenden Organmangels in Deutschland nicht gerecht. Es sind jedoch die intensivmedizinisch tätigen Ärzte, die die entscheidenden Gespräche über das Für und Wider der Zustimmung zur Spende mit den Angehörigen eines Patienten führen müssen, wenn dieser keine Willensäußerung abgegeben hat. Dieser Beitrag diskutiert verschiedene Probleme und Fragen, die sowohl in der Fachliteratur als auch im klinischen Alltag immer wieder thematisiert werden:

a) Der IHA und die Organspende sind seltene Ereignisse; so selten, dass sich in der Erkennung potenzieller Spenderinnen sowie der Einholung der Zustimmung durch die Angehörigen keine Routine ausbilden kann. Organspende muss, selbst wenn die eigentliche Spende selten ist, Normalität werden. Hilfestellung bei der Spendererkennung können z.B. automatisierte Abfragen in PDMS bieten. Die Bewertung dieser Informationen bleibt aber alleinige Aufgabe der verantwortlichen, behandelnden Intenisivmedizinerinnen und des TxB.

b) Ein Großteil der Bevölkerung möchte Organe spenden. Einer der Hauptgründe, eine Organspende abzulehnen, besteht in der Vorstellung, zu alt oder zu krankzu sein. Dabei sind tatsächliche medizinische Kontraindikationen selten. Es bestehen sowohl aufseiten der Ärztinnen als auch aufseiten der Patientinnen große Wissenslücken. Das Schließen dieser Lücken, z. B. über die
Vertrauensperson Hausarzt, könnte ein zusätzliches Spenderpotenzial mobilisieren.

c) Zumindest die 3 monotheistischen Weltreligionen erlauben eine Organspende.

d) Obwohl das Einführen einer Widerspruchslösung mit einem Anstieg der Spenderinnenraten assoziiert ist, erscheint der Effekt dieser Maßnahme jedoch marginal.

e) Die Frage nach PV und Vorsorgevollmachten gehört mittlerweile zum Standard im Aufnahmeprozedere nahezu jeder Klinik. Die Evaluation einer evtl. Organspendebereitschaft sollte ebenso normalisiert werden. Die Frage nach der Einstellung zur Organspende schafft Klarheit und Sicherheit im Umgang mit dem Thema. Die Beratung zum Thema Organspende, ggf. einschließlich des Anbietens von Informationsmaterial, erinnert sowohl Angehörige als auch Patientinnen daran, sich hiermit auseinanderzusetzen.

f) Muss eine Entscheidung zur Spende erst eingeholt werden, wenn die Patientin sie nicht mehr selbst äußern kann, wird sie oft aufgrund von Unwissenheit der Angehörigen über den genauen Willen der Patientin abgelehnt.

g) Patientenverfügungen beinhalten zu selten eindeutige Äußerungen zur Organspende. Grundsätzlich sind sich viele Autorinnen einig, dass die Fortführung einer Intensivtherapie über einen begrenzten Zeitraum von 48-72 $\mathrm{h}$ zur Ermöglichung einer Spende bei wirksamer Patientenverfügung legitim und richtig ist, da die Spende bei Zustimmung ebenfalls Ausdruck des zu respektierenden Patientenwillens ist.

h) Die ICOD ist in Deutschland als Konzept noch weitgehend unbekannt, sollte aber ins Blickfeld der Intensiv- und Notfallmedizinerinnen gerückt werden, da sie vom TPG abgedeckt wird.

i) Der Ausweitung des Spenderpools über den Hirntod hinaus im Sinne der CDCD muss eine intensive gesellschaftliche und politische Diskussion mit Auseinandersetzung zu den Themen Lebensende und Sterben vorausgehen. Vor der Diskussion konkreter Ideen zur Umsetzung muss eine gesamtgesellschaftlich akzeptierte, gesetzlich verankerte Position gefunden werden. 
Korrespondenzadresse

\section{Dr. A. Rand, DESAIC}

Klinik und Poliklinik für Anästhesiologie und Intensivtherapie, Universitätsklinikum Carl Gustav Carus

Fetscherstr. 74, 01307 Dresden, Deutschland Axel.rand@uniklinikum-dresden.de

\section{Einhaltung ethischer Richtlinien}

Interessenkonflikt. A. Rand, T. Koch und M. Ragaller geben an, dass kein Interessenkonflikt besteht.

Für diesen Beitrag wurden von den Autoren keine Studien an Menschen oder Tieren durchgeführt. Für die aufgeführten Studien gelten die jeweils dort angegebenen ethischen Richtlinien.

\section{Literatur}

1. Alaluf Ri (2020) Organ donation in judaism. Exp Clin Transplant 18:24-26. https://doi.org/10.6002/ ect.rlgnsymp2020.L4

2. Albar MA (1996) Islamic ethics of organ transplantation and brain death. Saudi J Kidney Dis Transplant 7:109-114

3. Arbeitspapier zum Verhältnis von Patientenverfügung und Organspendeerklärung. Dtsch Arztebl Int 110:572-574

4. Arshad A, Anderson B, Sharif A (2019) Comparison of organ donation and transplantation rates between opt-out and opt-in systems. Kidney Int 95:1453-1460. https://doi.org/10.1016/j.kint. 2019.01.036

5. Ashkenazi T, Steinberg A, Cohen J (2019) A national survey of attitudes of the Zionist ultra-orthodox community in Israel to organ donation. Prog Transplant Aliso Viejo Calif 29:43-47. https://doi. org/10.1177/1526924818817062

6. Blake J (2015) Religious beliefs and attitudes to organ donation. Welsh Government Social Research

7. Blum K (2012) Inhousekoordination bei Organspenden - Forschungsgutachten im Auftrag der Deutschen Stiftung Organtransplantation (DSO). Deutsches Krankenhausinstitute. V., Düsseldorf

8. Brauer M, Günther A, Pleul K et al (2019) Wie viele potenzielle Organspender gibt es wirklich?: Retrospektive Analysezu nichterfolgterDiagnostik des irreversiblen Hirnfunktionsausfalls bei verstorbenen Patienten mit relevanter Hirnschädigung. Anaesthesist 68:22-29. https://doi.org/10.1007/ s00101-018-0510-x

9. Caille-Brillet A-L, Zimmering R, Thaiss HM (2019) Bericht zur Repräsentativstudie 2018 „Wissen, Einstellung und Verhalten der Allgemeinbevölkerung zur Organ- und Gewebespende". Bundeszentrale für gesundheitliche Aufklärung (BZgA), Köln

10. Cypel M, Levvey B, Van Raemdonck D et al (2015) International society for heart and lung transplantation donation after circulatory death registry report. J Heart Lung Transplant 34:1278-1282. https://doi.org/10.1016/j.healun.2015.08.015

11. Domínguez-Gil B, Coll E, Elizalde J et al (2017) Expanding the donor pool through intensive care to facilitate organ donation: results of a Spanish multicenter study. Transplantation 101:e265-e272. https://doi.org/10.1097/TP.0000000000001701

\section{Organ donation-Not only a responsibility of intensive care medicine}

In 2019 a total of 756 people died in Germany while registered on the waiting list for an organ transplantation. With 10.8 organ donors/million inhabitants in 2019, Germany belongs to the bottom group in the Eurotransplant foundation as well as worldwide. All political attempts to increase the number of organ donations have so far been unsuccessful. Furthermore, the pandemic triggered by the severe acute respiratory syndrome coronavirus 2 (SARS-CoV-2) led to a further decline in organ donations. Critical care physicians play an important role in the identification of potential doners and are also the main point of contact for relatives; however, multiple uncertainties exist regarding the process of organ donation not only in discussions in the media and society but also among physicians involved in intensive care medicine. Many assumptions and hypotheses, which have been associated with the low number of donors, lack scientific evidence and are discussed in this article.

\section{Keywords}

Transplantation - Brain death - Circulatory death - Religious beliefs - Presumed consent
12. DSO (2019) Jahresbericht Organspende und Transplantation in Deutschland

13. English V (2007) Is presumed consent the answer to organ shortages? Yes. BMJ 334:1088-1088. https://doi.org/10.1136/bmj.39199.475301.AD

14. Eurotransplant International Foundation (2019) Annual Report 2019

15. Formanek M, Schöffski O (2010) Difficulties with the organ donation process in small hospitals in Germany. Transplant Proc 42:1445-1448. https:// doi.org/10.1016/j.transproceed.2010.01.067

16. Foss S, Nordheim E, Sørensen DW et al (2018) First Scandinavian protocol for controlled donation after circulatory death using normothermic regional perfusion. Transplant Direct 4:e366. https://doi.org/10.1097/TXD.0000000000000802

17. Gemeinsame Stellungnahme des Bevollmächtigten des Rates der EKD bei der Bundesrepublik Deutschland und der Europäischen Union und des Leiters des Kommissariats der deutschen Bischöfe - Katholisches Büro in Berlin - zu dem Gesetzentwurf von Jens Spahn et al. „Entwurf eines Gesetzes zur Regelung der doppelten Widerspruchslösung im Transplantationsgesetz" (BTDrs. 19/11096) und zu dem Gesetzentwurf von Annalena Baerbock et al. „Entwurf eines Gesetzes zur Stärkung der Entscheidungsbereitschaft bei der Organspende" (BT-Drs. 19/11087)

18. Gesetz über die Spende, Entnahme und Übertragung von Organen und Geweben (Transplantationsgesetz - TPG) zuletzt geändert durch Art. 16 G v. 19. Mai 202011018

19. Götze M (2020) Analyse des Organspenderpotentials der DSO Region Ost in den Jahren 2014-2016. Dissertation, Friedrich-Schiller-Universität Jena. https://doi.org/10.22032/DBT.40832

20. Heide W (2016) „Non-heart-beating donors" sind nicht geeignet. Nervenarzt 87:161-168. https:// doi.org/10.1007/s00115-015-0048-y

21. Hoisl A, Barbey R, Graf BM et al (2015) Wertungen des „Transplantationsskandals" durch die Medien: Diskursanalytische Studie an ausgesuchten deutschen Zeitungen. Anaesthesist 64:16-25. https://doi.org/10.1007/s00101-014-2406-8

22. Kootstra G, Daemen JH, Oomen AP (1995) Categories of non-heart-beating donors. Transplant Proc 27:2893-2894

23. Lomero M, Gardiner D, Coll E et al (2020) Donation after circulatory death today: an updated overview of the European landscape. Transpl Int 33:76-88. https://doi.org/10.1111/tri.13506

24. Manara A, Procaccio F, Domínguez-Gil B (2019) Expanding the pool of deceased organ donors: the ICU and beyond. Intensive Care Med 45:357-360. https://doi.org/10.1007/s00134-019-05546-9

25. Messer S, Page A, Axell R et al (2017) Outcome after heart transplantation from donation after circulatory-determined death donors. J Heart Lung Transplant 36:1311-1318. https://doi.org/ 10.1016/j.healun.2017.10.021

26. Miller FG, Truog RD, Brock DW (2010) The dead donor rule: can it withstand critical scrutiny? J Med Philos 35:299-312. https://doi.org/10.1093/jmp/ jhq019

27. Pross C, Berger E, Siegel M et al (2018) Stroke units, certification, and outcomes in German hospitals: a longitudinal study of patient-based 30-day mortality for 2006-2014. BMC Health Serv Res 18:880. https://doi.org/10.1186/s12913-0183664-y

28. Richtlinie gemäß $\S 16$ Abs. 1 S. 1 Nr. 3 TPG zur ärztlichen Beurteilung nach § 9a Abs. 2 Nr. 1 TPG (RL BÄK Spendererkennung). Dtsch Arztebl Int 117:A-1650

29. Richtlinie gemäß §16 Abs.1 S.1 Nr. 1 TPG für die Regeln zurFeststellung des Todes nach $\S 3$ Abs.1 S.1 Nr. 2TPG und die Verfahrensregeln zurFeststellung des endgültigen, nicht behebbaren Ausfalls der Gesamtfunktion des Großhirns, des Kleinhirns und des Hirnstamms nach $\S 3$ Abs. 2 Nr. 2 TPG, Vierte Fortschreibung. Bundesärztekammer

30. Rithalia A, McDaid C, Suekarran S et al (2009) Impact of presumed consent for organ donation on donation rates: a systematic review. BMJ 338:a3162-a3162. https://doi.org/10.1136/bmj. a3162

31. Schulte K, Borzikowsky C, Rahmel A et al (2018) Decline in organ donation in Germany. Dtsch Aerztebl Online. https://doi.org/10.3238/arztebl. 2018.0463

32. Tackmann E, Dettmer S (2019) Measures influencing post-mortem organ donation rates in Germany, the Netherlands, Spain and the UK: a systematic review. Anaesthesist 68:377-383. https://doi.org/10.1007/s00101-019-0600-4

33. Trabitzsch A, Pleul K, Barlinn K et al (2021) An automated electronic screening tool (DETECT) for the detection of potentially irreversible loss of 
brain function. Dtsch Arztebl Int. https://doi.org/ 10.3238/arztebl.m2021.0307

34. Weiss J, Elmer A, Mahíllo B et al (2018) Evolution of deceased organ donation activity versus efficiency overa 15-year period:an international comparison. Transplantation 102:1768-1778. https://doi.org/ 10.1097/TP.0000000000002226

35. Wesslau C, Grosse K, Krüger R et al (2007) How large is the organ donor potential in Germany? Results of an analysis of data collected on deceased with primary and secondary brain damage in intensive care unit from 2002 to 2005. Transpl Int 20:147-155. https://doi.org/10.1111/j.14322277.2006.00413.x

\section{Reinhard Larsen, Alexander Mathes \\ Pocket Guide Beatmung}

Reinhard Larsen, Alexander Mathes

Berlin, Heidelberg: Springer Verlag 4. Auflage, 345 S., 24 Abb., (ISBN: 978-3-66263439-4), Softcover 29,99 EUR, eBook

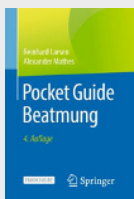

In der 4. Auflage des Pocket Guide Beatmung spannt das Autorenteam Reinhard Larsen und Alexander Mathes

den weiten Bogen der respiratorischen Unterstützungsverfahren von der SauerstoffInsufflation, über nicht-invasive und invasive Beatmungstechniken bis hin zur ECMOTherapie. Wer gemäß dem Titel ein taschenbuchartiges Nachschlagewerk erwartet, wird von dem Themenumfang und der Bandbreite des Buches sichtlich überrascht sein, muss aber dennoch nicht auf griffige und praxisnahe Empfehlungen, Checklisten und Standardeinstellungen für den klinischen Alltag verzichten.

Das Buch ist sehr strukturiert aufgebaut und führt den Leser zunächst von den pathophysiologischen Grundlagen der respiratorischen Insuffizienz äußerst übersichtlich durch die Nomenklatur der verschiedenen Beatmungsformen hin zur Indikationsstellung der Beatmungstherapie. Dabei ist den Autoren aber stets der klinische Blick auf den Patienten und die individuelle Entscheidungsfindung sowie die Definition eines Therapieziels wichtig: Die Beatmungstherapie wird nicht nur auf Beatmungsmodi, Geräteeinstellungen oder andere technische Aspekte reduziert, sondern stets als ein Therapiebaustein in einem intensivmedizinischen Gesamtkonzept gesehen. Daher werden Aspekten wie z.B. Physiotherapie, Weaning, Ernährung, Lagerungsbehandlung und Therapie der Grunderkrankung entsprechender Stellenwert und Platz im Buch eingeräumt.

Für typische respiratorische Krankheitsbilder wie COPD, ARDS, Thoraxtrauma etc. bekommt der Leser zudem übersichtliche Behandlungsempfehlungen im Checklistenformat. Ebenso werden Komplikationen der Beatmung und besondere Situationen, wie z.B. Transporte von Beatmungspatienten oder die Beatmung bei Schädel-Hirn-Trauma mit praxisnahen Tipps ausführlich behandelt. Separate Kapitel wie "Respiratorische Viruserkrankungen", "Palliative Beatmung" und
"Beatmung bei Kapazitätsengpässen" unterstreichen die Aktualität dieser 4. Auflage, die während der Corona-Pandemie entstanden ist.

Mit dem Pocket Guide Beatmung erhält der Leser eine komplette Übersicht von der Sauerstoff-Insufflation bis hin zur ECMOTherapie. Das Buch kann dabei sowohl als griffiges Nachschlagewerk für den klinischen Alltag dienen, als auch für die Rekapitulation der (Be-) Atmungsphysiologie. Die äußerst übersichtlichen Grafiken und die alltagsrelevanten Berechnungshilfen und Formeln helfen beim Verständnis der physiologischen, physikalischen und technischen Zusammenhänge.

Auch für den fortgeschrittenen Anwender von Beatmungstherapien bietet der Pocket Guide immer noch ausreichend Detailtiefe, wenn z.B. besondere Beatmungsformen, die automatische Tubuskompensation oder die transpulmonale Druckmessung erklärt werden. Wer dann noch weiter in komplexe Materie vordringen möchte erhält am Ende jedes Kapitels hilfreiche Literaturreferenzen.

A. Hohn (Mönchengladbach) 